\title{
Il regime mediatico in Italia: 1994-1995
}

Valérie Joelle Kouam

\section{OpenEdition}

\section{Journals}

Edizione digitale

URL: http://journals.openedition.org/cei/134

DOI: $10.4000 /$ cei. 134

ISSN: 2260-779X

Editore

UGA Éditions/Université Grenoble Alpes

\section{Edizione cartacea}

Data di pubblicazione: 15 juin 2010

Paginazione: 171-181

ISBN: 978-2-84310-168-7

ISSN: 1770-9571

\section{Notizia bibliografica digitale}

Valérie Joelle Kouam, «ll regime mediatico in Italia: 1994-1995», Cahiers d'études italiennes [Online], 11 | 2010, online dal 15 décembre 2011, consultato il 26 mars 2021. URL: http://journals.openedition.org/ cei/134; DOI: https://doi.org/10.4000/cei.134 


\title{
IL REGIME MEDIATICO IN ITALIA: 1994-1995
}

\author{
Valérie Joelle Kouam \\ Institut de Culture de Yaoundé
}

Indagare sul regime mediatico in Italia e sui cambiamenti decisivi dei mezzi di comunicazione avvenuti nella società italiana negli anni I994-1995 non è impresa facile ma, seppure in maniera sintetica, è lo scopo di questo lavoro. L'argomento risulta essere estremamente interessante sia nel suo aspetto storico che nelle sue componenti sociologiche che, inevitabilmente, vanno ad interagire con la realtà politica del Paese. E' sembrato interessante andare a verificare quale sia l'immagine datane alla luce della trasformazione del quadro politico italiano scaturito negli anni presi in considerazione. Naturalmente non potendo, per ragioni di tempo, scandagliare tutte le testate giornalistiche di quel periodo, si è valutato di analizzare solo uno. Si è quindi scelto di focalizzare l'attenzione sull'analisi di un unico quotidiano. La scelta è caduta su: La Voce. Perché?

Primo, perché La Voce vuole essere un giornale innovativo, alternativo ${ }^{\mathrm{I}}$, che non diventi mai una scarsa copia del telegiornale del giorno precedente. Secondo perché è un giornale creato da Montanelli dopo esser stato costretto ad abbandonare il quotidiano Il Giornale che egli stesso aveva fondato vent'anni prima. Il rapporto tra il giornalista di Fucecchio e quello che fu per diversi anni il suo editore (dal 1977, quando Berlusconi divenne socio di Il Giornale) era diventato conflittuale. In effetti, Montanelli si rifiutava di fare il megafono a Forza Italia. Inoltre si è scelto di focalizzare l'attenzione sugli anni 1994-1995 alla luce dell'evoluzione del quadro politico ed istituzionale dell'Italia.

Il periodo esaminato copre quindi gli anni dal I994 al I995: un lasso temporale che, sebbene troppo corto per fornire giudizi definitivi, risulti

I. Per raggiungere quest'obiettivo cè l'utilizzo di una grafica davvero innovativa che faccia concorrenza alla televisione e la scelta di poche importanti notizie da approfondire intensamente, lasciando le altre meno importanti relegate in spazio ridotto. 
estremamente suggestivo, se analizzato alla luce dei cambiamenti avvenuti nella società italiana.

Nella prima parte, quindi, dopo un'opportuna apertura sul contesto geopolitico, sui media e un breve ripasso storico, si passa in seguito ad analizzare gli articoli di Indro Montanelli usciti dal 1994 al 1995 su La Voce in riferimento al potere attribuito alle televisioni di Berlusconi. Ed anche la pubblicità o comunicazione persuasiva commerciale, la quale ha accompagnato in modo sempre più consistente e frequente, come tutti hanno potuto facilmente riscontrare, la quotidianità nel periodo preso in considerazione nel nostro lavoro.

\section{Il contesto geopolitico}

Quando parte ufficialmente la televisione in Italia, il 3 gennaio 1954, è, come nel resto d'Europa, una televisione pubblica gestita dallo Stato in regime di monopolio. In effetti, «in ciascun paese d'Europa, la televisione si era affermata negli anni Cinquanta-Sessanta come un servizio pubblico gestito direttamente o indirettamente dallo Stato, che rifletteva le specificità e i caratteri culturali di ognuno di loro" (Menduni, 2002, p. 25). Nonostante le numerose richieste eseguite nel corso degli anni da liberi cittadini intenzionati a gestire una propria emittente televisiva, il Parlamento si dimostra non favorevole e anche la Corte Costituzionale, interpellata a proposito, si pronuncia a sfavore. Solo dopo accese battaglie giudiziarie nel 1974 viene permessa la televisione privata. Prima relativamente alla televisione via cavo (in ambito locale) a seguito della Sentenza n. 225 del 1974 della Corte Costituzionale, e poi anche relativamente alla televisione terrestre (sempre in ambito locale) a seguito della Sentenza n. 202 del 1976 della Corte Costituzionale. A seguito di tale liberalizzazione nascono centinaia di emittenti televisive locali. Tra queste le emittenti del gruppo Fininvest primeggiano e si trasformano da realtà locali a realtà nazionali ottenendo prima l'autorizzazione a coprire tutta l'Italia, poi l'uso della diretta, prima di allora non concessa alle televisioni private. Nasce così una realtà aziendale, Mediaset ${ }^{2}$, in grado di rivaleggiare con il servizio pubblico della Rai.

2. Mediaset S.p.A. è un'azienda italiana specializzata in comunicazione televisiva, quotata alla Borsa italiana e controllata dalla holding Fininvest. E' stata fondata da Silvio Berlusconi nel 1978. Rappresenta il primo gruppo di network televisivi privati italiani (concorrente diretto della Rai), così denominato dal 1995 in seguito allo scorporo delle attività tv dalla finanziaria madre. La sede legale è a Milano, in via Paleocapa, 3, mentre la sede principale è a Cologno Monzese (Milano) in viale Europa. Possiede attività anche in Spagna, grazie al pos- 
La dimensione pubblica entra in quella privata tramite diverse tecnologie, vecchie e nuove, presenti nelle case, mentre molti aspetti della vita privata degli individui sono resi pubblici dai media. La televisione è un mezzo di comunicazione di massa, ossia uno strumento attraverso il quale è possibile indirizzare conoscenza verso una pluralità di destinatari indistinti. Alla televisione va posta una speciale attenzione. In effetti, negli anni 1994-1995, l'Italia è un paese con troppa televisione. In particolare, è la principale fonte informativa, perché solo una ridotta minoranza di persone legge libri e giornali (Istat, 1996). La televisione ha contribuito in modo non marginale a condizionare e a modificare il modo di vivere e di pensare. Si sa che la televisione è recepita dalla quasi totalità dei cittadini e concorre ad una prima scrematura e sistematizzazione degli innumerevoli eventi che formano il nostro vissuto collettivo, racconta eventi che sono ancora in corso e i cui sviluppi dipenderanno anche dal modo in cui verranno riferiti. La televisione, insomma, può non limitarsi ad influenzare un pubblico passivo, ma può essere in grado di provocare direttamente l'attualità di cui ha bisogno. La televisione è dunque capace di modificare strutture mentali e conseguenti modi di pensiero e comportamenti. Molto più di altri media la televisione è, oltre che veicolo di messaggi, lei stessa il messaggio (McLuhan, 199I). In più, negli anni 1994-I995, la tv pubblica è fortemente condizionata dalle maggioranze politiche. C'è da evidenziare la qualità di alcune trasmissioni, la messa in onda di queste in orari in grado di attirare grandi quantità di pubblico televisivo fa pensare a delle strategie comunicative di Berlusconi e del suo partito Forza Italia. Tutto nello scopo di accrescere o diminuire il prestigio, la fama, l'autorità di cui gode una persona, un gruppo, una associazione, un movimento o un partito politico, non solo per ciò che dicono di essi, ma anche per il semplice fatto di concedere molto spazio nei propri programmi. La comunicazione di massa ha accresciuto l'importanza dei tratti personali, quali l'aspetto, la voce, l'abito, il modo di parlare, come fattori nella vita sociale e politica.

Bisogna segnalare che in Italia non vi è stata, fino al 1992, alcuna regolamentazione dell'emittenza privata, per cui le reti commerciali hanno operato senza licenza per circa quindici anni, rischiando che, da un giorno all'altro, la magistratura ne sospendesse le trasmissioni. Con l'ovvia conseguenza che ogni imprenditore del settore, se ha potuto, ha cercato amici politici influenti in grado d'intervenire in caso di necessità.

sesso (attraverso la controllata Mediaset Investimenti) del 50,13\% di Telecinco (sempre fondata da Berlusconi nel I990 e ora primo canale spagnolo per ascolti) e di Endemol. Del Gruppo Mediaset fa parte anche Medusa Film dal 2007, società specializzata nella produzione e distribuzione di film italiani ed internazionali e nell'home entertainment. 
La scelta di Silvio Berlusconi di scendere in campo affiancando alla propria realtà d'imprenditore in grado di spartire con la televisione pubblica un vero e proprio duopolio, il suo ruolo di leader di un partito così integrato con l'impero delle comunicazioni è una situazione particolare che merita una certa attenzione. Non c'è dubbio che, a partire dalla campagna elettorale di Berlusconi nel 1994, la televisione sia progressivamente diventata un'arena centrale della politica italiana. Ma quest'arena è ancora largamente condizionata dalle logiche e dalle esigenze della politica. Non per niente, quando il leader di Forza Italia ha deciso di entrare in politica ha trasformato le proprie reti televisive in canali per diffondere le proprie pubblicità e i propri messaggi. Nasce così una situazione di dipendenza dal potere politico della televisione. Tra il 1994 e il I995, si ha un'influenza politica della televisione. Come noto, infatti, le caratteristiche e il successo della campagna elettorale di Berlusconi hanno indotto numerosi osservatori a interpretare la sua vittoria come un vero e proprio caso di videocrazia. Con questa parola, si intende il potere della televisione di condizionare le scelte politiche dei cittadini; oppure l'uso della televisione a tal fine (Cortelazzo, 1996). Il leader di Forza Italia ha usato la televisione come canale per la politica, cioè l'ha trasformata in una tribuna dalla quale rendere visibile e lanciare un nuovo prodotto in un mercato politico improvvisamente destrutturato. Un ulteriore elemento di cui tener conto è il ruolo chiave giocato dalle sue televisioni sulle quali, soprattutto nella fase iniziale della sua storia, si è appoggiato Berlusconi. Delle strutture assai potenti per connettere organizzazione e interessi. Si vede il potere del mezzo televisivo.

Ci si pùo fare la domanda seguente: il successo di Berlusconi potrebbe essere interpretato come una vittoria dei mezzi di comunicazione? Per provare a documentare più efficacemente quest'ipotesi, in seguito discuteremo i principali cambiamenti analizzando le rappresentazioni proposte da Indro Montanelli ne La Voce.

\section{Commenti ed interviste su La Voce}

Le elezioni del '94 hanno fatto epoca: un caso così unico come quello di Berlusconi ha segnato un punto di svolta nel dibattito sul rapporto politica-televisione in Italia. Nel suo giornale La Voce ${ }^{3}$, Montanelli mette

3. La Voce sorse idealmente dalle ceneri di Il Giornale, anch'esso creato da Indro Montanelli e da lui abbandonato dopo vent'anni di pubblicazioni, lamentando pesanti ingerenze direttamente da Silvio Berlusconi, che 
in guardia dall'enorme potere dei media e dice: «oggi per instaurare un regime, [...] Bastano i cosidetti mezzi di comunicazione di massa: e fra essi, sovrano e irresistibile, la televisione.» (Montanelli, Io ottobre 1994.) In effetti, negli anni presi in considerazione, un grande spazio è stato dato nei palinsesti al dibattito, alla discussione, al confronto. La tv ha avuto un suo ruolo e il leader del partito Forza Italia se ne è servito: «Man mano che la politica entrava in crisi cercava nell'uso della TV un riparo» (Follini, 1994). Montanelli parla della televisione come mezzo usato da Berlusconi: "che trova soprattutto nel video la propria arena» (Montanelli, I8 giugno 1994). In effetti, il successo elettorale del 27 marzo 1994 ridà ossigeno al gruppo Fininvest e la successiva quotazione in borsa dell'azienda, con la nascita di Mediaset, risolvono i problemi finanziari del gruppo, ma non quelli giudiziari. Per lui: «delle sei grandi reti a diffusione nazionale, le tre private sono proprietà del capo del governo; le tre pubbliche sono state affidata a dirigenti nominativi da un consiglio d'amministrazione a sua volta nominato da autorità rappresentanti delle forze governative, le quali non fecero mistero della loro intenzione di mettere la tv al servizio del Potere» (Montanelli, I5 novembre 1994). E Berlusconi diventa monopolista televisivo assoluto. Le tv Fininvest sono sue: «il nodo della Fininvest sono i suoi tre telegiornali coi quali si può manipolare come si vuole la pubblica opinione, senza dimenticare quelle Rai le controlla grazie al potere politico» (Montanelli, I5 novembre 1994). Montanelli dice a proposito delle televisioni di Berlusconi: «uno strazio aggiuntivo di questi torridi giorni sono per me le apparizioni sul video del Cavaliere che avendone a disposizione sei tra pubbliche e privati, non perde occasioni di abusarne» (Montanelli, 22 luglio 1994). E per lui il Cavaliere «dispone in esclusiva dello strumento principe di manipolazione della pubblica opinione: la tv, sia privata che di Stato" (Montanelli, 20 settembre 1994). Il giornalista toscano continua sottolineando il fatto che Berlusconi sia molto presente alla televisione: «da quattro mesi non riusciamo a liberarci di Berlusconi che dal video c'invade la casa a pranzo, a cena e a letto, sembra proprio il contrario» (Montanelli, 30 luglio 1994). A volte, Montanelli sembra anche scandalizzato e fa paragoni: «io credo che in quarant'anni di potere Andreotti abbia occupato le televisioni meno di quanto il Cavaliere abbia fatto in quattro mesi» (Montanelli, 30 luglio 1994). Montanelli suona la sirene d'allarme:

auspicava una netta presa di posizione da parte della testata in favore della sua ascesa politica. Tali pressioni furono dal direttore respinte e la sua permanenza a $\mathrm{Il}$ Giornale non fu più possibile. Così il giornalista fiorentino, all'età di ottantacinque anni, ricomincia con una nuova testata, affermando la sua libertà e indipendenza, e le sue posizioni. Questo giornale nasce alla vigilia del primo governo Berlusconi. I 385 giorni di vita de La Voce hanno inciso l'informazione italiana. 
«sei reti televisive (tre Rai e tre Fininvest) che, accantonati dibattiti e risse, intonino l'osanna al nuovo regime ed al suo «timoniere» (Montanelli, 9 giugno 1994).

A causa dell'anomalità della situazione che regola i mezzi di comunicazione e la stessa informazione, arrivano decreto e legge che dovrebbero porre un freno allo strapotere della Fininvest. Montanelli critica le scelte fatte. Dice agli Italiani: «firmate, fermateli, dopo la decisione del governo di cancellare con decreto la carcerazione preventiva per cittadini accusati di reati gravissimi: corruzione, concussione, truffa, droga» (Montanelli, 26 luglio 1994). Cominciano le leggi tese a tutelare i suoi interessi privati. Montanelli ne riassume alcuni. Usa un tono dubitativo piuttosto ironico: «Forse non sarà vero (se ne dicono tante) che egli controlli, oltre a quelli dichiaratamente suoi, alcuni giornali e riviste che gli fanno da reggicoda. [...] I suoi mezzi di comunicazione - e soprattutto le televisioni - sono alla mercé di killer che contro i nemici - o ritenuti tali - di Berlusconi vomitano regolarmente accuse ed insulti da codice penale» (Montanelli, I2 aprile 1994). Berlusconi ha imposto i suoi temi programmatici, che sono stati i soli dibattuti nella campagna elettorale. Ha anche focalizzato il dibattito sulla crisi economica, sul fisco e sull'occupazione (il famigerato milione di posti di lavoro). La sinistra non ha fatto altro che ridicolizzare le proposte di Berlusconi, senza suggerirne altre di più convincenti. Il Cavaliere, oltretutto, scendendo in campo, ha posto all'ordine del giorno la sua legittimazione a fare il leader, riducendo il dibattito politico solo al problema se lui meritasse o meno la fiducia degli italiani. Ecco la soluzione da Montanelli raccomandata: «il problema della Fininvest si risolve in un modo solo: con la vendita. [...] Comunque, irrinunciabile rimane il principio: interesse pubblico e interesse privato non sono miscelabili: o si serve l'uno, o si serve l'altro" (Montanelli, 30 luglio 1994).

L'opinione pubblica in quel periodo si divideva sulla vita di Berlusconi: c'era chi gli ricordava l'amicizia con Craxi, la sua ambigua ascesa economica, il conflitto d'interessi e c'era invece chi lo ammirava per la sua bravura di imprenditore e la capacità di essersi «fatto da solo». Cavaliere nero o nomo del miracolo? Essendo così impostata, per larga parte, l'informazione politica dal fatidico 26 gennaio, è stata indirizzata l'opinione pubblica a decidere se Berlusconi fosse «buono» o "cattivo» e non tanto se votare per il polo di destra, di sinistra o di centro.

Nel '94 la Fininvest sviluppa una precisa strategia di marketing: rigonfia di politica i palinsesti di reti caratterizzate e targettizzate come Rete 4 e Italia $I$ in modo da costruire una nuova comunicazione politica indirizzata al pubblico giovanile senza dimenticare quello femminile. Se il 
servizio pubblico mostra di rivolgersi a tutti in generale, il Polo sembra privilegiare, nella sua comunicazione, un pubblico specifico ${ }^{4}$. Questa specifica strategia di marketing appare ispirata a fini politici piuttosto che commerciali. Come spiegare altrimenti la presenza di programmi come $O$ di qua o di là? e la nascita di ben 7 trasmissioni targate Fininvest (Braccio di ferro; Dieci domande a; Elettorando; Funari leader; Luogo comune; Qui Italia; $O$ di qua o di là) durante la campagna elettorale o comunque in prossimità della stessa?

Per non parlare poi di quello che è successo negli ultimi cinque giorni di campagna, le reti Fininvest hanno aumentato l'attenzione per il Polo delle Libertà di più del $15 \%$. La Rai ha avuto, ed ha, un ruolo istituzionale che non le ha permesso di schierarsi apertamente; la Fininvest, invece, potendosi muovere più liberamente nell'informazione politica, non ha avuto paura di promuovere eccessivamente un partito.

Montanelli attraverso La Voce racconta esaurientemente l'offerta televisiva e trova che l'ascesa e affermazione politica di un partito come Forza Italia e di un leader come Berlusconi, (che a nostro parere rappresentano sotto molti punti di vista un'anomalia nel panorama politico internazionale, data l'inestricabile commistione fra interessi pubblici e privati che attraverso di essa si realizza), sono state favorite, anche se non certo unilateralmente determinate, dalle innovative strategie di comunicazione politica adottate nel 1994 e anche dopo. Secondo Montanelli la televisione ha avuto molta influenza in quanto: «l'impatto della stampa sulla pubblica opinione è quello del ronzio di una zanzara nella grande orchestra di una televisione massicciamente schierata - anche quella pubblica - dalla parte del governo e intesa a silenziare, ignorandola, qualsiasi voce contraria» (Montanelli, 30 luglio 1994).

Durante tutta la campagna elettorale Berlusconi è stato sicuramente il vincitore della corsa all'immagine, totalizzando oltre cinque ore e mezza di intervento in diretta nelle trasmissioni giornalistiche di intrattenimento. A sentire Montanelli, Berlusconi ha vinto perché ha saputo presentarsi meglio, ha sfoggiato la migliore immagine di sé. Egli analizza le strategie comunicative di Forza Italia e del suo leader Silvio Berlusconi, fondatore di un partito che ha fatto significativamente la sua prima comparsa

4. Ad esempio Rete 4 si rivolge in modo particolare ad un pubblico femminile, con età superiore ai 50 anni, con un livello d'istruzione prevalentemente inferiore e riferibile ad una modesta condizione socioeconomica: una tipologia di pubblico da telenovelas. Anche Italia I si caratterizza per un pubblico specifico, quello di età media, i giovani: le sue trasmissioni detengono infatti i valori più alti tra il pubblico che va dai 6 ai 30 anni. E' chiaro che la strategia della Fininvest era di coinvolgere nel dibattito politico un pubblico che fino ad allora ne era più o meno rimasto estraneo, con un'offerta di «telepolitica» semplificata e a volte «spettacolarizzata». 
nel panorama politico italiano con un discorso preregistrato diffuso attraverso una videocassetta il 26 gennaio del 1994. Ha saputo presentarsi come «il nuovo», nonostante la sua evidente occupazione dello spazio politico lasciato libero dalla caduta del vecchio pentapartito travolto dagli scandali di Tangentopoli. Dopo appena due mesi dalla sua nascita, è riuscito a conquistare una quantità impressionante di consensi, diventando inaspettatamente il primo partito italiano col $2 \mathrm{I} \%$ dei voti espressi.

Lo spazio offerto dalle televisioni a Berlusconi è tanto, per questo: «A differenza dei politici suoi predecessori che vivevano solo di politica» (Montanelli, Io settembre 1994). Berlusconi è un caso a parte in quanto si è abituato «a trattare il Paese come una audience, di cui contano solo gl'indici di ascolto e di gradimento» (Montanelli ıo settembre 1994). Grazie anche ad una massiccia campagna elettorale, le elezioni politiche del 27 marzo 1994 si concludono con un successo del suo partito in corsa con Alleanza Nazionale di Fini e con la Lega Nord di Bossi. Negli ultimi mesi di campagna elettorale, alcuni fra i volti più famosi's delle reti Fininvest dichiarano in televisione il loro appoggio politico, all'interno dei programmi di intrattenimento da loro condotti, scatenando reazioni che scaturiscono nella discussa par condicio. La prima esperienza di governo di Silvio Berlusconi ha però vita dura e breve, e si conclude nel dicembre dello stesso anno, quando la Lega Nord ritira l'appoggio al Governo. Il 22 dicembre Berlusconi rassegna le proprie dimissioni al presidente della Repubblica Oscar Luigi Scalfaro. All'inizio, si potrebbe attribuire la responsabilità della caduta del governo di Berlusconi all'inaffidabilità di Bossi. In seguito invece la si potrebbe piuttosto attribuire alla magistratura e a Scalfaro, il quale, secondo lo stesso Berlusconi, avrebbe indotto Bossi a ritirare l'appoggio all'esecutivo. Polo e Lega si riconcilieranno in occasione delle elezioni politiche del 200 .

In Italia, l'ascesa al potere e al controllo, pressoché completo, delle comunicazioni di massa, televisive e giornalistiche, del personaggio Silvio Berlusconi, già criticato per le modalità di accumulazione della sua fortuna imprenditoriale grazie ad amicizie politiche ed oscuri rapporti con persone e organizzazioni poco raccomandabili, ha determinato una situazione che si configura come una vera e propria dittatura mediatica, rispetto alla quale persino il Capo dello Stato, Carlo Azeglio Ciampi, è stato indotto a segnalare in un messaggio alle Camere:

5. Tra questi Mike Bongiorno, Raimondo Vianello e Sandra Mondaini, Ambra Angiolini ed Iva Zanicchi che in un programma d'intrattenimento della domenica pomeriggio dichiara che la sua mamma voterà per Silvio "perché i ricchi, essendosi già arricchiti, non avrebbero interesse a rubare dalle tasche dei cittadini». 
nel nostro paese rispetto all'articolo 2I della Costituzione repubblicana e a tutti i principi di pluralismo che caratterizzano la Carta del 1948. L'unica risposta che è venuta, in oltre un anno, dalla maggioranza parlamentare e dal Governo che fanno capo all'attuale presidente del Consiglio Berlusconi, è stato il varo del disegno di legge Gasparri per il riassetto del sistema radiotelevisivo, che sta per essere approvato in maniera definitiva dal Parlamento e che condurrà, senza dubbio alcuno, al consolidarsi dell'attuale oligopolio televisivo con l'aggravante della depressione delle risorse pubblicitarie per la Rai e l'ulteriore espansione di Mediaset e, dunque, del potere mediatico detenuto, insieme con il controllo della pubblicità complessiva, dall'uomo di Arcore. Non c'è dunque da sperare, almeno in tempi brevi e a meno di un improbabile crollo della maggioranza raccolta intorno alla Casa delle Libertà, che le cose possano cambiare e che gli italiani riacquistino il proprio elementare diritto all'informazione, pur facendo esso parte di quelli fondamentali stabiliti dal dettato costituzionale.

Il governo Berlusconi è caduto a dicembre del 1994 con le dimissioni del suo primo ministro, che aveva ricevuto in novembre un avviso di garanzia da parte della procura di Milano. Infuriavano le polemiche sull'opportunità di mettere sotto inchiesta il Presidente del Consiglio. Il I3 gennaio del 1995 il Presidente della Repubblica Oscar Luigi Scalfaro ha affidato a Lamberto Dini l'incarico di formare il nuovo governo. Montanelli sembrava felice e si è subito augurato che non si comporti come Berlusconi, cioè tentare: "di ammannirci dal video, mane e sera, la sua immagine» (Montanelli, I4 gennaio 1995).

Il famigerato decreto legge "per la parità d'accesso ai mezzi d'informazione durante le campagne elettorali», ben noto come par condicio ${ }^{6}$ ha avuto un finto effetto nella realtà italiana. In effetti, gli art. 2, 5, 6, 9 di questo decreto impongono dei limiti che sembrano molto vaghi. Questi limiti imposti ai giornalisti televisivi possono benissimo essere sviati. Sappiamo che il giornalista può esercitare la sua influenza in mille modi. La valutazione non è facile da fare. Comunque questo decreto stimola il dibattito sull'obiettività e sulle regole dell'informazione.

Durante il mese d'aprile 1995, il clima politico e sociale è surriscaldato. Ci sono le elezioni amministrative nelle quali 43 milioni di elettori andranno a votare per rinnovare le amministrazioni di 15 regioni, 75 province, 5 II 9 comuni. Berlusconi spera di poter vincere e convincere in questo modo ad andare subito alle politiche, ma Montanelli lo prende in giro: «Inutile chiedergli quale testo di legge o sentenza di Alta Corte stabilisca tale consecutio: per lui questi non sono problemi.» (Montanelli, 2 aprile 1995.) Montanelli pensa già allo scenario in caso di vittoria di

6. Decreto legge n. 83 del 20 marzo I995. 
Berlusconi. Non gli piacerebbe perché sarebbe «immediatamente riecheggiata dai mezzi d'informazione targati Fininvest, da cui dobbiamo prepararci ad essere martellati i prossimi giorni» (Montanelli, 2 aprile 1995).

Un anno e venti giorni dopo il suo primo numero, il I2 aprile I995, La Voce cessò le pubblicazioni.

\section{Conclusione}

Il repertorio degli scontri che hanno continuato a susseguirsi negli anni I994-I995 fra i media, il leader politico Silvio Berlusconi e il quadro dei rapporti fra media e politica sin qui analizzato confermano una certa crisi che sembra abbia investito il sistema della comunicazione politica italiana negli anni presi in considerazione nel nostro lavoro. Per Montanelli, l'evolversi della storia in questa direzione può indurre a una stanca rassegnazione con la conseguenza di arrivare a concepire la politica quale mera gestione dell'esistente, quale cosa banale: «Berlusconi. Sempre Berlusconi. Solo Berlsuconi. Per otto mesi l'Italia è stata (anche per gli stranieri, ahimè) Berlusconi. Per otto mesi non si è potuto intavolare, nemmeno in famiglia, una conversazione che non avesse per argomento Berlusconi o non ci finisse.» (Montanelli, 23 dicembre 1994.) Inoltre, possiamo affermare che sia difficile pensare che una propaganda diretta e massiccia possa da sola orientare l'opinione pubblica. Ma una piccola parte di elettorato può effettivamente essere orientato dalla TV e, nell'era del maggioritario, questo piccolo numero può essere determinante. A questo proposito concludiamo dicendo che la televisione ha avuto un potere notevole, ovvero ha influenzato in qualche modo gli italiani. Come riferito da Ilvo Diamanti: "Tutto si è giocato, dunque, attraverso il ricorso alla televisione: quelle nazionali ancor di più di quelli locali» (Diamanti, 1994). Le televisioni e i giornali del Presidente del Consiglio sono stati efficienti. Lungi da ogni allarmismo, possiamo affermare che il fatto che il capo di un governo possegga anche il monopolio dell'informazione, ha segnato la storia italiana. 


\section{Bibliografia}

Cortelazzo Michele, Annali del Lessico Contemporaneo Italiano. Neologismi 1995, Padova, Esedra, 1996.

Diamanti Ilvo et Mannheimer Renato, Milano a Roma: guide all'Italia elettorale del 1994, Rome, Donzelli editore, 1994.

Follini Marco, «La supplenza della televisione», I problemi dell'Informazione, n. 3, 1994.

Istat, Cultura socialità tempo libero, Indagine Multiscopo sulle famiglie. Anni 1993-1994, Roma, Istat, 1996.

La Repubblica del 24 aprile 1995.

McLuhan Marshall, La Galassia Gutenberg. Nascita dell'uomo tipografico, Roma, Armando editore, I99I.

—, Gli strumenti del comunicare, Milano, Il Saggiatore, 1967.

Menduni Enrico, Televisione e società italiana 1975-2000, Milano, Bompiani, 2002.

Montanelli Indro, «Per favore Cavaliere», La Voce del I2 aprile I994.

—, "Ma non sarà un bluff-trust?", La Voce del 30 luglio I994.

—, "In nome della gente», La Voce del 9 giugno 1994.

- La Voce del I8 giugno I994.

—, La Voce del 22 luglio 1994.

—, "Quando il lettore alza la voce», La Voce del 26 luglio 1994.

—, La Voce del io settembre 1994.

—, «La Strage degl'innocui», La Voce del 20 settembre 1994.

—, "La fabbrica del regime», La Voce del Io ottobre 1994.

—, «Servi, padroni e pari condizioni», La Voce del I5 novembre 1994.

—, "Il tramonto dei gladiatori», La Voce del I4 gennaio 1995.

—, "Il polo delle bugie», La Voce del I9 gennaio 1995 .

—, "Che ci azzeccano gli ebrei?», La Voce del 2I gennaio 1995.

—, "Vola colomba bianca vola», La Voce del 24 gennaio 1995.

—, "Grazie lettori», La Voce del 29 marzo 1995.

—, "C'era una volta un re», La Voce del 2 aprile 1995.

—, "Uno straniero in Italia», La Voce del I2 aprile 1995.

—, "C'era una volta un re», La Voce del 2 aprile 1995.

Morcellini Mario, Elezioni di Tv, Genova, Costa \& Nolan, 1995. 
